\title{
Nonisothermal Multicomponent Reactive Transport Model for Unsaturated Soil
}

\author{
Suresh Channarayapatna Seetharam¹; Hywel Rhys Thomas²; and Philip James Vardon ${ }^{3}$
}

\begin{abstract}
A multicomponent reactive transport model, coupled with an existing thermal, hydraulic, and mechanical model for porous media, is investigated. The model is based on conservation of mass/energy principles for the flow and stress-strain equilibrium for the mechanical behavior. The resultant model is coupled with a geochemical model to capture geochemical interactions. Numerically, the Galerkin FEM is employed for spatial discretization and an implicit Euler method for temporal discretization. The coupling of the transport and geochemical models is achieved through both noniterative and iterative approaches. A series of applications are considered to demonstrate the numerical performance and qualitative behavior, specifically in the context of multicomponent behavior. The model shows good convergence and computational efficiency. DOI: 10.1061/(ASCE)GM.1943-5622.0000018. @ 2011 American Society of Civil Engineers.
\end{abstract}

CE Database subject headings: Computer analysis; Transport phenomena; Geotechnical models; Coupling; Unsaturated flow; Unsaturated soils; Chemicals.

Author keywords: Models; Computer analysis; Transport phenomena; Geotechnical models; Coupling; Unsaturated flow; Unsaturated soils; Chemicals.

\section{Introduction}

A number of multicomponent reactive chemical transport models with varying capabilities have been proposed in recent years. Some of the models include the effect of saturated and variably saturated soil conditions (e.g., Yeh and Tripathi 1991), some consider nonisothermal behavior (e.g., Steefel and Lasaga 1994), and a few have recently attempted to incorporate the effect of soil deformation (e.g., Guimarães et al. 2006; Cleall et al. 2007a). These developments have led to the so-called coupled thermalhydraulic-chemical-mechanical (THCM) models. Such models are applied in the field of geoenvironmental engineering in problems such as contaminant transport, geothermal engineering, underground nuclear waste repository, and landfill designs. The relevance of multicomponent chemicals in these applications is that a more complete description of soil chemical behavior can be achieved.

Recent work by the writers and coworkers has led to the development of a comprehensive THCM model based on a mechanistic approach (Cleall et al. 2007a, b), formed into a computational code named COMPASS. The model essentially extends an existing coupled thermal-hydraulic-mechanical model for unsaturated soil

\footnotetext{
${ }^{1}$ Research Fellow, Geoenvironmental Research Centre, Cardiff School of Engineering, Cardiff Univ., Cardiff, UK. E-mail: seetharams@ cardiff.ac.uk

${ }^{2}$ Professor and Director, Geoenvironmental Research Centre, Cardiff School of Engineering, Cardiff Univ., Cardiff, UK (corresponding author). E-mail: thomashr@cardiff.ac.uk

${ }^{3}$ Research Fellow, Geoenvironmental Research Centre, Cardiff School of Engineering, Cardiff Univ., Cardiff, UK. E-mail: vardonpj@cardiff .ac.uk

Note. This manuscript was submitted on July 1, 2009; approved on April 5, 2010; published online on April 10, 2010. Discussion period open until September 1, 2011; separate discussions must be submitted for individual papers. This paper is part of the International Journal of Geomechanics, Vol. 11, No. 2, April 1, 2011. CASCE, ISSN 1532-3641/2011/ $2-84-89 / \$ 25.00$.
}

to include a multicomponent reactive transport model. To account for geochemical interactions, a geochemical model has been coupled with the multicomponent transport model. Such coupled models offer several numerical challenges, such as stability, convergence, speed of computation, and the need to handle a large number of degrees of freedom with varying magnitudes. The writers recently carried out some work to investigate these numerical aspects of the THCM model (Seetharam et al. 2007). In particular, numerical implementation was considered in detail, with specific emphasis on the multicomponent aspects such as generation of finite-element matrices, handling of sink/source terms, geochemical coupling technique, and time-step control. Through a number of examples of varying degrees of complexity, both qualitative behavior and numerical performance, such as convergence and computational efficiency, were examined. In this paper, these numerical aspects are further explored.

\section{Governing Equations}

In terms of soil behavior, the following processes can be recognized: (1) chemical transport through advection, diffusion (molecular and thermal), mechanical dispersion, geochemical reactions; (2) hydraulic transport through vapor flow (diffusive and advective flow), liquid flow (pressure gradient and osmotic gradient); (3) dry air flow through diffusion and pressure gradient; (4) thermal transfer by conduction, convection, and latent heat of vaporization; and (5) mechanical deformation in swelling soils. The geochemical reactions are solved via a geochemical model, MINTEQA2. Only the final form of the governing equations for these processes will be presented here as the detailed formulation has been documented elsewhere (Thomas et al. 1998; Cleall et al. 2007a). The equations are expressed in five primary variables, namely, pore water pressure $\left(u_{l}\right)$, pore air pressure $\left(u_{a}\right)$, temperature $(T)$, total dissolved concentration $\left(c_{d}^{i}\right)$, and displacements $\mathbf{u}$ (in $x, y$, and $z$ directions). 
Multicomponent chemical transfer:

$$
\begin{aligned}
C_{c_{d} l}^{i} \frac{\partial u_{l}}{\partial t}+C_{c_{d} a}^{i} \frac{\partial u_{a}}{\partial t}+C_{c_{d} T}^{i} \frac{\partial T}{\partial t}+C_{c_{d} c_{d}}^{i} \frac{\partial c_{d}^{i}}{\partial t}+C_{c_{d} s}^{i} \frac{\partial s_{s}^{i}}{\partial t}+C_{c_{d} u}^{i} \cdot \frac{\partial \mathbf{u}}{\partial t} \\
=\nabla \cdot\left[K_{c_{d} l}^{i} \nabla u_{l}\right]+\nabla \cdot\left[K_{c_{d} T}^{i} \nabla T\right]+\nabla \cdot\left[K_{c_{d} c_{d}}^{i} \nabla c_{d}^{i}\right] \\
\quad+\nabla \cdot\left[\sum_{j=1}^{n c,(j \notin i)} K_{c_{d} c_{d}}^{j} \nabla c_{d}^{j}\right]+J_{c_{d}}^{i}
\end{aligned}
$$

Moisture transfer

$$
\begin{aligned}
C_{l l} \frac{\partial u_{l}}{\partial t}+C_{l T} \frac{\partial T}{\partial t}+C_{l a} \frac{\partial u_{a}}{\partial t}+C_{l u} \cdot \frac{\partial \mathbf{u}}{\partial t} \\
=\nabla \cdot\left[K_{l l} \nabla u_{l}\right]+\nabla \cdot\left[K_{l T} \nabla T\right]+\nabla \cdot\left[K_{l a} \nabla u_{a}\right] \\
\quad+\nabla \cdot \sum_{j=1}^{n c} \rho_{l} K_{l_{c_{d}}}^{j} \nabla c_{d}^{j}+J_{l}
\end{aligned}
$$

Heat transfer

$$
\begin{gathered}
C_{T l} \frac{\partial u_{l}}{\partial t}+C_{T T} \frac{\partial T}{\partial t}+C_{T a} \frac{\partial u_{a}}{\partial t}+\sum_{i=1}^{n c} C_{T c_{d}^{i}} \frac{\partial c_{d}^{i}}{\partial t}+C_{T u} \cdot \frac{\partial \mathbf{u}}{\partial t} \\
=\nabla\left[K_{T l} \nabla u_{l}\right]+\nabla\left[K_{T T} \nabla T\right]+\nabla\left[K_{T a} \nabla u_{a}\right]+\sum_{i=1}^{n c} K_{T c_{d}^{i}} \nabla c_{d}^{i} \\
+\sum_{i=1}^{n c} V_{T c_{d}^{i}} \nabla c_{d}^{i}+V_{T l} \nabla u_{l}+V_{T T} \nabla T+V_{T a} \nabla u_{a}+J_{T}
\end{gathered}
$$

Dry air transfer

$$
\begin{gathered}
C_{T l} \frac{\partial u_{l}}{\partial t}+C_{T T} \frac{\partial T}{\partial t}+C_{T a} \frac{\partial u_{a}}{\partial t}+\sum_{i=1}^{n c} C_{T c_{d}^{i}} \frac{\partial c_{d}^{i}}{\partial t}+C_{T u} \cdot \frac{\partial \mathbf{u}}{\partial t} \\
=\nabla\left[K_{T l} \nabla u_{l}\right]+\nabla\left[K_{T T} \nabla T\right]+\nabla\left[K_{T a} \nabla u_{a}\right]+\sum_{i=1}^{n c} K_{T c_{d}^{i}} \nabla c_{d}^{i} \\
+\sum_{i=1}^{n c} V_{T c_{d}^{i}} \nabla c_{d}^{i}+V_{T l} \nabla u_{l}+V_{T T} \nabla T+V_{T a} \nabla u_{a}+J_{T}
\end{gathered}
$$

Deformation

$$
C_{u l} d u_{l}+C_{u T} d T+C_{u a} d u_{a}+C_{u u} d \mathbf{u}-\mathbf{P} . \mathbf{D}_{e p} \varepsilon_{s}^{p}+d \mathbf{b}=0
$$

where $C_{p q}=$ storage coefficients; $K_{p q}$ and $V_{p q}=$ flux coefficients; and $J_{p}=$ gravity terms. For example, $C_{c_{d} l}^{i}$ represents chemical storage coefficient affected by change in the liquid storage term (pore-water pressure); $C_{l T}$ represents the liquid storage affected by the change in temperature, for example, because of the change in density of water vapor at thermodynamic equilibrium; and $J_{L}$ represents liquid flow caused by gravity. The details of these coefficients can be found in Thomas et al. (1998) and Cleall et al. (2007a). The deformation equation is cast in an incremental form. The term $\rho_{l}$ in Eq. (2) represents density of water and the terms $\mathbf{P}$, $\mathbf{D}_{e p}, \varepsilon_{s}^{p}$, and $\mathbf{b}$ in Eq. (5) represents strain matrix, elastoplastic coefficient matrix, strain vector, and body force vector, respectively. The term $s_{s}^{i}$ in Eq. (1) represents sink/source for the multicomponent chemicals. This term is evaluated via the geochemical model, MINTEQA2, and represents sorbed and/or precipitated quantity of chemical species. The theoretical aspects of the MINTEQA2 model can be found in Allison et al. (1991).

The proposed model is capable of addressing generic reactive transport of multicomponent chemicals under nonisothermal, saturated/unsaturated conditions for small strain deformation problems and specifically where a chemical equilibrium condition is applicable. Some applications that can be considered are studies of coupled thermo/hydro/chemical/mechanical behavior of bentonite material in nuclear waste disposal concepts and multicomponent contaminant transport in the subsurface.

\section{Numerical Approach}

Two different schemes are adopted to solve the above set of governing equations. For spatial discretisation, the Galerkin FEM is employed, whereas a fully implicit finite-difference scheme is employed for temporal discretisation (Zienkiewicz and Taylor 1989). These methods are summarized in the following section.

\section{Spatial Discretization}

The basic conservation equations relating to Eqs. (1)-(4) can be generalized as

$$
\frac{\partial M}{\partial t}+\nabla \cdot \underline{q}=0
$$

where $M=$ mass or enthalpy; and $q=$ flux term and can be discretized by using the Galerkin method as

$$
\int_{\Omega}\left(N_{m}^{t} \frac{\partial M}{\partial t}+\nabla N_{m}^{t} \cdot \underline{q}\right) d \Omega+\int_{\Gamma_{1}} N_{m}^{t} \cdot \underline{q}^{*} d \Gamma=0
$$

where $q *=$ flux prescribed at boundary $\Gamma_{1} ; N_{m}=$ shape function; $N_{m}^{t}=$ transpose of the shape function; and $\Omega=$ domain. Similarly, for the deformation Eq. (5) combined with the use of a shape function $N_{f}$, yields

$$
\begin{aligned}
& \int_{\Omega}\left(C_{u l} d u_{l}+C_{u T} d T+C_{u a} d u_{a}+C_{u u} d \mathbf{u}\right) d \Omega \\
& \quad-\int_{\Gamma_{1}} N_{f}^{t}\left(\nabla d u_{a}+d \mathbf{b}_{\mathbf{i}}\right) d \Omega-\int_{\Gamma_{1}} N_{f}^{t} \underline{\tau} d \Gamma \\
& \quad=0
\end{aligned}
$$

where $\underline{\tau}=$ surface traction.

The preceding formulation permits the use of various boundary conditions such as Dirichlet, Neumann, and Cauchy. The spatially discretized equations can then be expressed as

$$
\underline{A} \underline{\phi}+\underline{B} \frac{\partial \underline{\phi}}{\partial t}+\underline{C}=\{0\}
$$

where $\phi, \underline{A}, \underline{B}$, and $\underline{C}=$ matrices of the global unknowns, the coefficient $\mathbf{K}$ matrix, coefficient $\mathbf{C}$ matrix, and flux vectors, respectively. Detailed implementation of the numerical scheme can be found in Seetharam (2003).

\section{Temporal Discretization}

Following a fully implicit midinterval forward difference timestepping scheme, a variant of the Euler method, the global unknowns can be determined through

$$
\underline{\phi}^{s+1}=\left[\underline{A}^{s+(1 / 2)}+\frac{\underline{B}^{s+(1 / 2)}}{\Delta t}\right]^{-1}\left[\frac{\underline{B}^{s+(1 / 2)} \underline{\phi}^{s}}{\Delta t}-\underline{C}^{s+(1 / 2)}\right]
$$

where $\phi^{s+1}=$ value of the unknowns at the new time step; and the superscripts $s$ and $s+(1 / 2)=$ sampling point at the previous time step and at the midinterval, respectively. Although the implicit 
scheme is unconditionally stable, there is a need for time-step control as in some problems large time steps can cause slow rate of convergence and large truncation/approximation errors. In particular, because of nonlinear material parameters, the approximation of sampling at time $s+(1 / 2)$ for the coefficients can lead to slow convergence. To address this, a variable time-stepping scheme is introduced. If the number of iterations required to meet convergence criteria exceeds a specified maximum, the time-step size is decreased by a factor, typically 1.05 . However, if the number of iterations is lower than a specified minimum, then the time-step size is increased by the same factor. Detailed implementation of the numerical scheme can be found in Seetharam (2003).

\section{Coupling of Transport and Geochemical Reaction Equations}

Two types of approaches are implemented to allow the coupling of transport and geochemical models, namely, a sequential noniterative approach (SNIA) and a sequential iterative approach (SIA) (e.g., Walter et al. 1994; Yeh and Tripathi 1989). In both the approaches the coupled flow (thermal, hydraulic, and chemical) and deformation equations are solved first followed by geochemical reactions. Essentially, nodal values of temperature and chemical concentrations (in molalities) are passed on to MINTEQA2, which in turn returns equilibrated values of chemical concentrations. These new concentrations would form an input to either next iteration or next time step as appropriate. To couple the COMPASS program with MINTEQA2, minor modifications to the latter program are necessary to ensure efficient transfer of data between the two programs without writing values to disk. To accelerate computation, provision is made to impose a tolerance check, in other words, if the concentrations of chemicals and the temperature do not vary more than a specified amount, say $0.01 \%$, then the program skips the geochemical analysis for that particular node. The detailed implementation of the two coupling schemes can be found in Seetharam et al. (2007).

\section{Application}

To demonstrate the numerical capability of the coupled model, a series of pseudo one-dimensional (1D) horizontal axisymmetric problem have been considered. Verification and validation of the proposed model are separately dealt with by Cleall et al. (2007a, b). The material is assumed to be a highly swelling clay such as a bentonite. The domain has an internal diameter of $0.525 \mathrm{~m}$, an external diameter of $0.815 \mathrm{~m}$, a height of $0.01 \mathrm{~m}$, and is discretized into 128 eight-noded quadrilateral elements. An initial time step of $7 \mathrm{~s}$ is considered, which is allowed to increase to a maximum of 0.5 days, depending on the convergence rate. The total duration of the simulation is set to 70 days, as trial runs show that the hypothetical specimen becomes saturated during this time period. For instance, depending on the size of laboratory specimen and initial state, it may take several weeks to several months for full saturation of bentonite material (e.g., Huertas et al. 2000). An LU solver is used for solving the matrices. The SIA coupling technique is employed for all problems presented subsequently because the SNIA can in some cases display large errors (e.g., Yeh and Tripathi 1991), especially when heterogeneous reactions are involved. Therefore, while numerical convergence may be reached with the SNIA method, systematic errors can cause potentially large errors in the macroscopic results.

The problem considers 12 degrees of freedom per node, i.e., pore-water pressure, temperature, displacements $(\mathrm{dr}, \mathrm{dz})$, and eight chemical components: $\mathrm{H}^{+}, \mathrm{Na}^{+}, \mathrm{K}^{+}, \mathrm{Ca}^{2+}, \mathrm{Mg}^{2+}, \mathrm{Cl}^{-}, \mathrm{SO}_{4}^{2-}$, and $\mathrm{CO}_{3}^{2-}$. The series of examples include (1) multicomponent transport with precipitation/dissolution reactions involving calcite, anhydrite, and halite; (2) same as Example (1) but with ion-exchange reactions between $\mathrm{Na}^{+}, \mathrm{K}^{+}, \mathrm{Ca}^{2+}$, and $\mathrm{Mg}^{2+}$; (3) same as Example (2) but with the addition of thermal gradient; and (4) same as Example (3) but with nonlinear elastic mechanical behavior.

For all problems, the domain is considered to be initially unsaturated with $55 \%$ degree of saturation (corresponding to a suction of $150 \mathrm{MPa}$ ) and with initial uniform pore-water chemical concentration, precipitate concentration, and ion-exchange complexes. The initial pore-water concentrations are different for separate examples because of the nature of geochemical reactions considered. For Example (1), the initial concentrations are as follows: $\mathrm{H}^{+}=0.29$, $\mathrm{Na}^{+}=1.679, \quad \mathrm{Ca}^{2+}=2.465, \quad \mathrm{Mg}^{2+}=0.027, \quad \mathrm{Cl}^{-}=1.57$, $\mathrm{SO}_{4}^{2-}=2.549, \quad \mathrm{CO}_{3}^{2-}=0.267, \quad \mathrm{CaCO}_{3}(s)=0.14, \quad$ and $\mathrm{CaSO}_{4}(s)=0.04 \mathrm{mmol} / \mathrm{kg}$. For Examples (2) to (4), the initial concentrations are as follows: $\mathrm{H}^{+}=0.69, \mathrm{Na}^{+}=39.14$, $\mathrm{K}^{+}=0.32, \mathrm{Ca}^{2+}=1.206, \mathrm{Mg}^{2+}=0.38, \mathrm{Cl}^{-}=1.57, \mathrm{SO}_{4}^{2-}=$ 20.33, $\quad \mathrm{CO}_{3}^{2-}=0.66, \quad \mathrm{CaCO}_{3}(s)=0.14, \quad \mathrm{CaSO}_{4}(s)=0.022$, $\mathrm{NaX}=0.57, \quad \mathrm{KX}=0.0064, \quad \mathrm{CaX}_{2}=0.067, \quad$ and $\mathrm{MgX}_{2}=$ $0.0203 \mathrm{mmol} / \mathrm{kg}$. An initial uniform temperature of $293 \mathrm{~K}$ is assumed for all examples. For Example (5), the soil is assumed to be initially in mechanical equilibrium with net mean stress of $500 \mathrm{kPa}$.

The hydraulic boundary conditions are same for all examples with hydration water supplied at a pressure of $1 \mathrm{MPa}$ on the right-hand side boundary, i.e., at $r=0.815 \mathrm{~m}$, which is referred to as the hydration surface. The hydration water consists of the same chemical components as in the initial pore water but at different concentrations as follows: $\mathrm{H}^{+}=1.23, \mathrm{Na}^{+}=42.78$, $\mathrm{K}^{+}=1.43, \quad \mathrm{Ca}^{2+}=2.66, \quad \mathrm{Mg}^{2+}=1.9, \quad \mathrm{Cl}^{-}=40.5, \quad \mathrm{SO}_{4}^{2-}=$ 6.075 , and $\mathrm{CO}_{3}^{2-}=1.3 \mathrm{mmol} / \mathrm{kg}$.

For Example (3), additionally, a constant temperature of $323 \mathrm{~K}$ is applied at the left boundary (at $r=0.525 \mathrm{~m}$ ), referred to as the hot surface, and a constant temperature of $293 \mathrm{~K}$ is applied at the hydration surface. For Example (4), in addition to thermal and hydraulic boundary conditions previously described, the domain is constrained at both the ends and is allowed to deform in the radial direction only.

The soil properties are assumed to be similar to MX-80 bentonite and are primarily obtained from Huertas et al. (2000). As far as geochemical properties such as thermodynamic equilibrium constants $\left(K_{e q}\right)$ are concerned, default MINTEQA2 database is used for geochemical speciation and precipitation/dissolution reactions. However, for ion-exchange reactions, the following equilibrium constants have been assumed: $K_{\mathrm{Na}-\mathrm{Ca}}=0.21, K_{\mathrm{NaK}}=0.26$, and $K_{\mathrm{Na}-\mathrm{Mg} 2+}=0.13$. Full details of material parameters and constants can be found in Seetharam et al. (2007).

\section{Qualitative Behavior}

Only salient results pertaining to anhydrite precipitation/ dissolution, sodium $(\mathrm{NaX})$ and calcium $\left(\mathrm{CaX}_{2}\right)$ ion-exchange behavior and void ratio and radial stress behavior will be presented in this section. Fig. 1 shows the anhydrite precipitation/dissolution behavior by comparing Examples (1) and (2). The precipitation/ dissolution behavior is predicted by the geochemical model on the basis of the total analytical concentrations of $\mathrm{Ca}^{2+}$ and $\mathrm{SO}_{4}^{2-}$ ions and the thermodynamic equilibrium constant relevant to this reaction. In both the cases, advancement of dissolution front is seen because of hydration. The quantitative difference is 


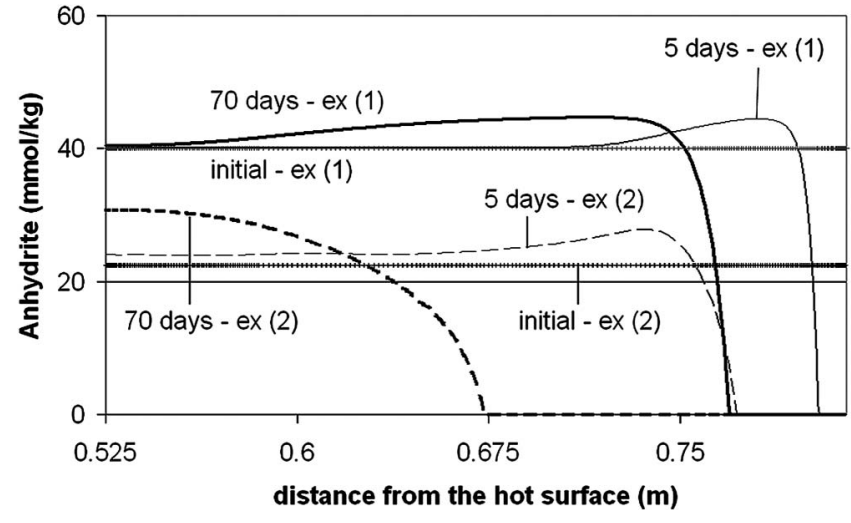

Fig. 1. Anhydrite precipitation/dissolution behavior for Examples (1) and (2)

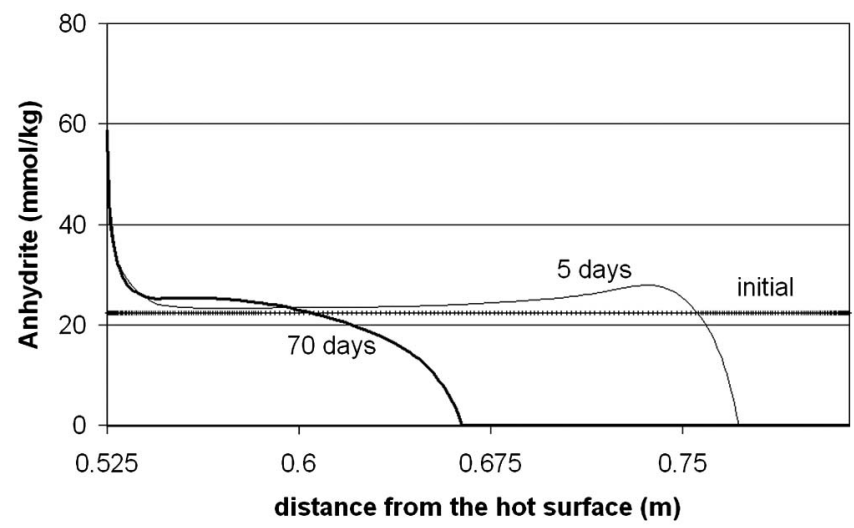

Fig. 2. Anhydrite precipitation/dissolution behavior for Example (3)

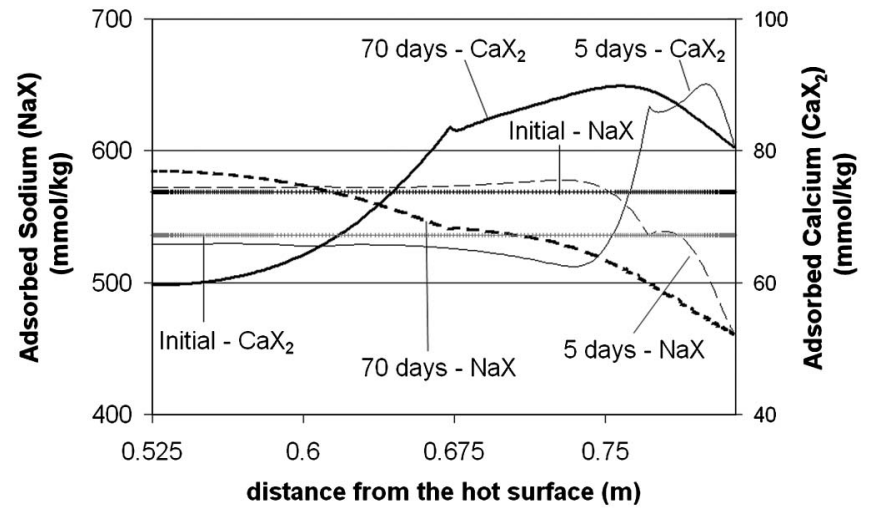

Fig. 3. Adsorbed $\mathrm{Na}^{+}$and $\mathrm{Ca}^{2+}$ behavior for Example (2)

attributed to the additional geochemical process of ion exchange in Example (2), which essentially affects the concentrations of $\mathrm{Ca}^{2+}$ and $\mathrm{SO}_{4}^{2-}$ ions.

Fig. 2 shows similar behavior as Example (2), however, there is an increased precipitation at the hot surface which is attributed to drying and the temperature effect on the solubility constant. Figs. 3 and 4 show the $\mathrm{Na}^{+}$and $\mathrm{Ca}^{2+}$ ion-exchange behavior for Examples (2) and (3), respectively. At the hydration surface, the adsorbed calcium $\left(\mathrm{CaX}_{2}\right)$ concentration increases while adsorbed sodium $(\mathrm{NaX})$ decreases. This is caused by the increase in dissolved $\mathrm{Ca}^{2+}$ concentration in the pore water because of the dissolution of anhydrite.

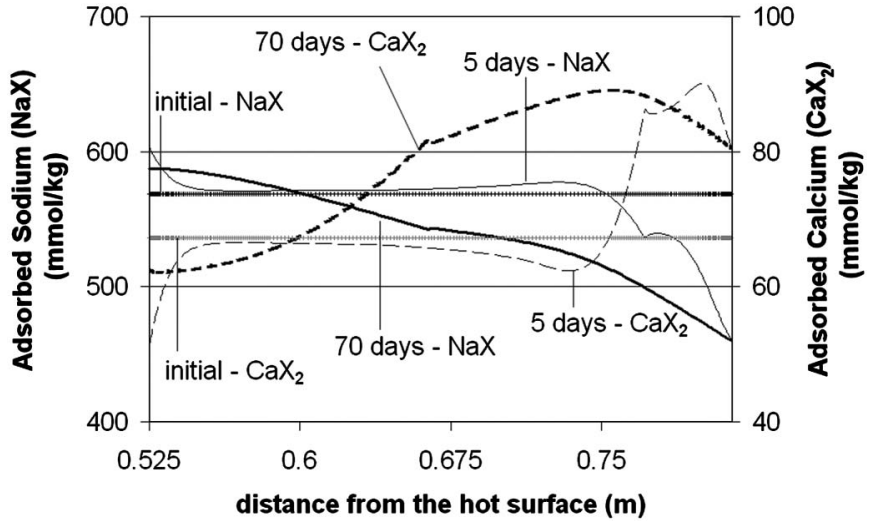

Fig. 4. Adsorbed $\mathrm{Na}^{+}$and $\mathrm{Ca}^{2+}$ behavior for Example (3)

Consequently, $\mathrm{Ca}^{2+}$ competes for adsorbed sodium ( $\mathrm{NaX}$ ) position. Fig. 4 shows similar behavior for Example (3) at the hydration surface, however, some interesting results are obtained near the hot surface that show a peak increase in adsorbed sodium $(\mathrm{NaX})$ and a corresponding decrease in adsorbed calcium $\left(\mathrm{CaX}_{2}\right)$ concentration. This is attributed to the increased precipitation of anhydrite at the hot end, which causes depletion of $\mathrm{Ca}^{2+}$ ions in the pore water, and hence, to achieve stoichiometric balance, dissolved $\mathrm{Na}^{+}$exchanges with adsorbed $\mathrm{Ca}^{2+}$. In conclusion, the model has provided results that are qualitatively consistent with the behavior of real systems.

\section{Numerical Aspects}

From a numerical point of view, the results presented in the "Qualitative Behavior" section demonstrated that the coupling between the temperature, moisture, multicomponent chemicals, displacements, and the following components of the geochemical model has been correctly implemented (qualitatively acceptable behavior): ion-exchange model, precipitation/dissolution, aqueous speciation, and the temperature correction model. Examples of verifications and validations of the proposed model can be found in Cleall et al. (2007a, b).

Pertaining to the preceding analysis, the following additional numerical aspects are highlighted:

Example (1): The numerical scheme has provided a stable solution and fast convergence (average of three iterations per time step). Some small computational efficiency gain has been achieved by introducing a tolerance check of $0.01 \%$ in concentration before the geochemical aspect of the analysis is undertaken. Approximately $60 \%$ of the nodes are skipped from the geochemical analysis during the first 55 time steps, and thereafter there is a gradual decrease in the number of nodes being skipped with none skipped beyond the 228th time step of the total 356 steps. This is because with time, the changes in the multicomponent behavior extend to the whole domain, and hence, nodes cannot be skipped.

Example (2): Once again, the numerical algorithm has provided stable solution and fast convergence has been achieved (average of three iterations per time step). However, in this example, the computational efficiency caused by the tolerance check has not been substantial. This is because of additional chemical processes, i.e., ion-exchange reactions, which bring about changes in most parts of the domain from the initial stages.

Example (3): Although the problem introduced an additional degree of freedom, i.e., temperature, the numerical characteristics were found to be similar to Example (2) in convergence and computational efficiency. 
Example (4): Although the mechanical model introduces highly nonlinear equation, the numerical characteristics are found to be similar to Example (1) in convergence, with some small computational efficiency achieved by introducing the tolerance check. Nearly $70 \%$ of the nodes are skipped from the geochemical analysis during the first 130 time steps, and thereafter there is a rapid decrease in the number of nodes being skipped with none skipped beyond 154th time step of the total 472 time steps.

Overall, the model showed good convergence for all time steps, although variable time-step sizes were necessary to avoid initial convergence problems. This is because initially the system is not in local equilibrium, and hence, more numbers of iterations are needed to achieve convergence. There were minor oscillation problems primarily during initial stages of the analysis. The tolerance check introduced to limit the need to carry out geochemical analysis yielded a small reduction in computational time that was attributed to the small size of the problem. However, it should be noted that for large problems in which the order of magnitude is in excess of 100,000 nodes, the time savings could be significant. As far as the geochemical model is concerned, fast convergence was achieved for every run, i.e., less than 20 iterations. Although not shown, no differences in the solution were found if nodes were not to be skipped.

One of the key challenges faced when dealing with large spatial and temporal problems is in solving large matrices in which large variations in the order of magnitude of the primary variables exist. For instance, in Example (4), pore-water pressure varies between $-90 \mathrm{MPa}$ and $0 \mathrm{MPa}$ whereas displacements vary between $10^{-5}$ and $10^{-7} \mathrm{~m}$. This can lead to ill-conditioned matrices, however, in all the analyses considered, the use of LU solver did not pose any significant problem given the small number of elements considered in this analysis.

\section{Computational Challenges}

The computation challenges related to the large spatial scales and timescales have been addressed by the application of domain decomposition and parallel algorithmic/computing techniques (Owen 2000; Thomas et al. 2003; Vardon et al. 2009). Although not presented in this paper, asymmetric compatible variants of the conjugate gradient solver, the biconjugate gradient (Bi-CG), conjugate gradient squared (CGS), and biconjugate gradient stabilized (Bi-CG STAB) methods have been implemented for nonlinear fully coupled problems providing significant time savings in this work. Use of such variants is necessary because of the nonsymmetric nature of the coupled governing equations considered. Preconditioning is performed by using Jacobi, or incomplete LU (ILU) decomposition based on a Crout factorization with an incompleteness of fill-in degree zero. In addition, when considering large spatial scales, such iterative solvers always maintain matrix sparsity, reducing computational storage requirements. Moreover, when considering large spatial or temporal scales, the time taken for the execution of simulations may be significant. High-performance computing (HPC), in this case parallel computation, may be used to increase the amount of computation undertaken at any one time.

Presently there are a number of computational architectures used for parallel processing. In general terms, these are shared memory, in which a number of processors access a single memory; distributed memory, in which each processor has access to a dedicated memory; and more recently, a combination of the two forms, in which a series of shared-memory nodes are connected in the same manner as a distributed-memory machine. Reflecting the computational architecture is the parallel programming paradigms of multithreaded models, in which a single program splits into many threads that can execute on other processors but must have access to the same memory; and message-passing, in which a number of programs execute separately, usually the same program running multiple times, and pass data through an interconnect when required. The shared-memory/multithreaded model usually outperforms the distributed-memory/message-passing model by virtue of avoiding data communication, but is restricted by the amount of processing cores available, on most HPC machines. It is shown by Vardon et al. (2008) that on modern HPC machines using message-passing to parallelize the Bi-CG solver yielded only limited computational gain and communication times dominated the solver operation, especially when using more than 8 processing cores.

To overcome this problem, a hybrid multithreaded/messagepassing form of the Bi-CG solver was implemented for use on nodal HPC machines, whereby the Krylov subspace, the most computational intensive part of the algorithm, was calculated using all available processing cores by using a multithreaded model on nodes and using message-passing across nodes. Less computationally intensive calculations, such as vector-vector multiplications, were undertaken by using only the multithreaded model on individual nodes. This model restricts the amount of communication undertaken, therefore boosting the computation-communication ratio. Up to a six times speed-up has been found when using 32 processing cores for problems consisting of approximately 500,000 degrees of freedom. More details and a comprehensive analysis of the computational performance can be found in Vardon et al. (2011)

\section{Conclusions}

This paper has considered some numerical aspects of the multicomponent reactive transport model. The model was based on a mechanistic approach. A brief overview of the theoretical formulation, including that of thermal, hydraulic, multicomponent chemical, and mechanical models was presented. The numerical implementation of the resulting system of governing equations was presented, and the Galerkin FEM and implicit Euler methods were discussed. The coupling of the resultant flow and deformation model to the geochemical model was briefly considered, which included noniterative and iterative approaches. A series of examples were then formulated in an increasing order of complexity, which demonstrated both the numerical performance and the overall capability of the model to predict the qualitative behavior consistent with real behavior. Some aspects of computational demands and challenges were explored. From the numerical perspective, the model provided a stable solution, good convergence, and some improved computational efficiency.

\section{Acknowledgments}

The writers would like to kindly acknowledge Dr. Peter Cleall for his contribution during early stages of this work.

\section{References}

Allison, J. D., Brown, D. S., and Novo-Gradac, K. J. (1991). MINTEQA2 user manual version 3.0, Environmental Research Laboratory, U.S. EPA, Athens, GA.

Cleall, P. J., Seetharam, S. C., and Thomas, H. R. (2007a). "Inclusion of some aspects of chemical behavior of unsaturated soil in thermo/hydro/ 
chemical/mechanical models. I: Model development." J. Eng. Mech., 133(3), 338-347.

Cleall, P. J., Seetharam, S. C., and Thomas, H. R. (2007b). "Inclusion of some aspects of chemical behavior of unsaturated soil in thermo/hydro/ chemical/mechanical models. II: Application and transport of soluble salts in compacted bentonite." J. Eng. Mech., 133(3): 348-356.

Guimarães, L. D. N., Gens, A., and Olivella, S. (2006). "Coupled thermohydro-mechanical and chemical analysis of expansive clay subjected to heating and hydration." Transp. Porous Media, 66(3), 341-372.

Huertas, F., et al. (2000). "Full-scale engineered barriers experiment for a deep geological repository for high-level radioactive waste in crystalline host rock (FEBEX project)." Final Rep. EUR 19147 EN, European Commission, Brussels, Belgium.

Owen, D. H. (2000). "Preconditioned parallel iterative solution methods for coupled finite element analyses." Ph.D. thesis, Cardiff Univ., UK.

Seetharam, S. C. (2003). "An investigation of the thermo/hydro/chemical/ mechanical behaviour of unsaturated soils." Ph.D. thesis, Cardiff School of Engineering, UK.

Seetharam, S. C., Thomas, H. R., and Cleall, P. J. (2007). "Coupled thermo/ hydro/chemical/mechanical model for unsaturated soils-Numerical algorithm.” Int. J. Numer. Methods Eng., 70(12), 1480-1511.

Steefel, C. I., and Lasaga, A. C. (1994). "A coupled model for transport of multiple chemical species and kinetic precipitation/dissolution reactions with application to reactive flow in single phase hydrothermal systems." Am. J. Sci., 294, 529-592.

Thomas, H. R., He, Y., and Onofrei, C. (1998). "An examination of the validation of a model of the hydro/thermo/mechanical behaviour of engineered clay barriers." Int. J. Numer. Anal. Meth. Geomech., 22(1), 49-71.
Thomas, H. R., Yang, H. T., He, Y., and Cleall, P. J. (2003). “A multi-level parallelised substructuring frontal solution for coupled thermo/hydro/ mechanical problems in unsaturated soil." Int. J. Numer. Anal. Meth. Geomech., 27, 951-965.

Yeh, G. T., and Tripathi, V. S. (1989). "A critical evaluation of recent developments in hydrogeochemical transport models of reactive multichemical components." Water Resour. Res., 25(1), 93-108.

Yeh, G. T., and Tripathi, V. S. (1991). "A model for simulating transport of reactive multispecies components: Model development and demonstration." Water Resour. Res., 27(12), 3075-3094.

Vardon, P. J., Banicescu, I., Cleall, P. J., Thomas, H. R., and Philp, R. N. (2009). "Coupled thermo-hydro-mechanical modelling: A new parallel approach." Proc., PDSEC-09 Workshop of the IEEE IPDPS 2009 Conf., Rome.

Vardon, P. J., Cleall, P. J., Thomas, H. R., and Philp, R. (2008). "Threedimensional field-scale coupled thermo-hydro-mechanical modelling." Proc., 12th Int. Conf. of the Int. Association for Computer Methods and Advances in Geomechanics, Goa, India.

Vardon, P. J., Cleall, P. J., Thomas, H. R., Philp, N., and Banicescu, I. (2011). "Three-dimensional field-scale coupled thermo-hydromechanical modeling: A parallel computing implementation." Int. J. Geomech., 11(2), 90-98.

Walter, A. L., Frind, E. O., Blowes, D. W., Ptacek, C. J., and Molson, J. W. (1994). "Modelling of multicomponent reactive transport in groundwater: Model development and evaluation." Water Resour. Res., 30(11), 3137-3148.

Zienkiewicz, O. C., and Taylor, R. L. (1989). The finite element method, 4th Ed. McGraw Hill, London. 